Case Report

\title{
Rare case of paracaecal hernia with intussusception presenting as small bowel obstruction: case report
}

\author{
M. S. Kalyan Kumar*, Shyamsundar R., Sabari Girieasan M., R. Kannan, S. Nedunchezhiyan
}

Department of Surgery, Madras Medical College, Chennai, Tamil Nadu, India

Received: 28 January 2020

Revised: 16 March 2020

Accepted: 18 March 2020

*Correspondence:

Dr. M. S. Kalyan Kumar,

E-mail: drkalyanms@gmail.com

Copyright: (c) the author(s), publisher and licensee Medip Academy. This is an open-access article distributed under the terms of the Creative Commons Attribution Non-Commercial License, which permits unrestricted non-commercial use, distribution, and reproduction in any medium, provided the original work is properly cited.

\begin{abstract}
Primary internal hernias are very rare in adults. They are an unusual cause of small intestinal obstruction and lead to high morbidity and mortality if left untreated. Clinical presentation of internal hernia is highly nonspecific. Imaging has limited role in diagnosing the cause of acute intestinal obstruction. Internal hernias are usually detected at laparotomy. We report a case of a 61-year-old male who presented with acute intestinal obstruction which was attributed later to a very rare type of internal hernia on exploratory laparotomy. A loop of ileum was found to enter the retroperitoneum through a hernia gate which was located lateral to the caecum and ileo ileal intussusception was noted proximal to the herniated loop. The segment of intestine was resected and anastamosed then hernial defect was closed. Paracaecal hernias are the rare type of hernias in internal hernia. In our case, intussusception was noted proximal to the herniated ileal loop which is a very rare presentation. Intussusception was reported previously with paraduodenal type. One should always keep in mind while conducting emergency laparotomy, internal hernias can be a cause for intestinal obstruction.
\end{abstract}

Keywords: Internal hernia, Intussusception, Paracaecal hernia, Small bowel obstruction

\section{INTRODUCTION}

An internal hernia (IH) is an infrequent cause of small bowel obstruction (SBO), with a reported autopsy incidence of $0.2-0.9 \%$, and is the cause of small-bowel obstruction in $0.6-5.8 \%$ of the cases. Preoperative diagnosis of internal hernia is extremely difficult because of the nonspecific clinical presentation. Abdominal computed tomography $(\mathrm{CT})$ plays an important role in the evaluation and management of patients with SBO. Urgent surgical intervention to prevent strangulation is essential. ${ }^{1}$

\section{CASE REPORT}

61 year old male patient who is a known diabetic and hypertensive for the past 20 years and 10 years respectively came with the complaints of diffuse abdominal pain for 4 days. He had vomiting for the past 2 days which was feculent. History of obstipation was present for past 3 days. No history of jaundice, hematemesis, melena, abdominal distension, fever, trauma, LOW, LOA. He was diagnosed to have pulmonary TB 2 years back and completed ATT CAT 1. He was a known case of CAD and on regular medication. $\mathrm{He}$ is not a smoker but alcoholic for the past 20 years. His GC was fair. BP $150 / 90$ and pulse 118/min. On examination abdomen was normal in shape. All quadrants moves equally with respiration. Soft, diffuse tenderness and no guarding or rigidity. No mass palpable. Liver dullness not obliterated. BS and exaggerated. Per rectal examination with normal fecal staining. Blood investigations were normal and viral markers were found to be negative. Cardiologist, medicine and diabetologist opinion were obtained. 
USG abdomen revealed dilated small bowel loops (3.2 $\mathrm{cm})$ with to and fro motion noted. No free fluid noted. CECT abdomen showed dilated small bowel loops noted with max diameter $3.1 \mathrm{~cm}$. large bowel loops and distal ileal loops collapsed. Evidence of telescoping of distal ileo-ileal loop noted with proximal dilated ileal and jejunum loops with multiple air fluid levels noted. Suggestive of ileo-ileal intussusception with small bowel obstruction. Hence patient was taken for emergency exploratory laparotomy.

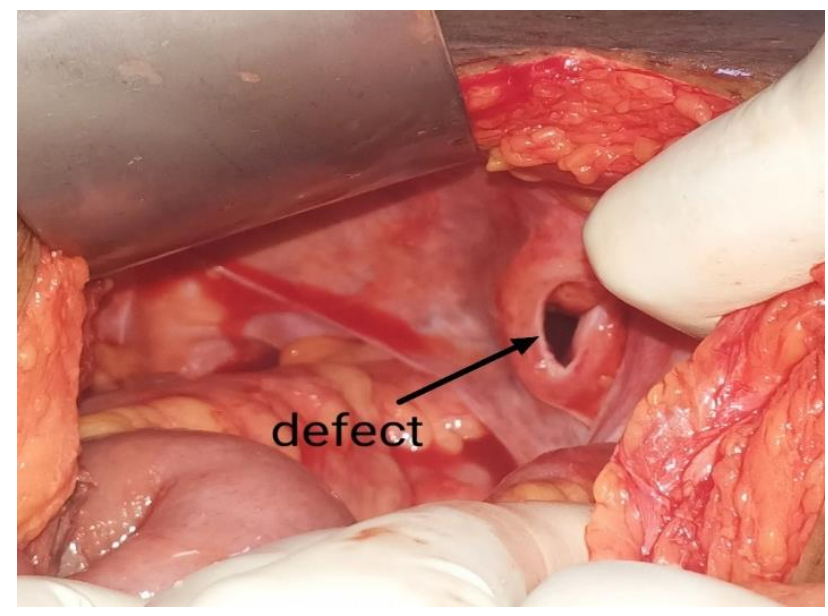

Figure 1: Paracaecal defect.

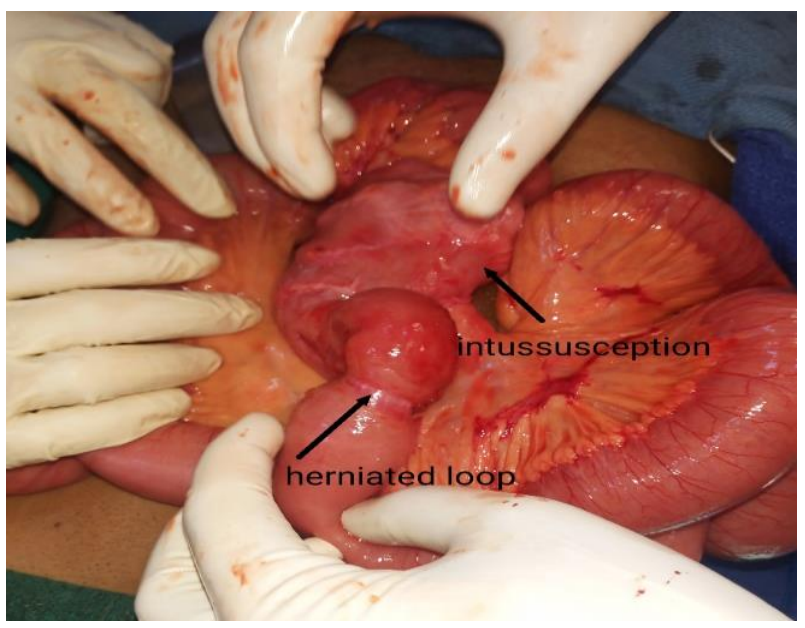

Figure 2: Herniated loop.

Intra operative findings were: a loop of distal ileum 15 $\mathrm{cm}$ from ileo caecal junction found to be herniating through the reflected part of peritoneum adjacent to caecum (Figure 1), proximal to the herniated ileal loop, ileo- ileal intussusception (Figure 3) was noted for about $15 \mathrm{~cm}$ length (Figure 2 and 4) and Omental adhesions were noted and released. Hence herniated ileal loop reduced manually and proceeded with resection of $20 \mathrm{~cm}$ segment and anastomosis (Figure 5) leaving $20 \mathrm{~cm}$ proximal to ileo caecal junction. Defect was closed primarily with 1-0 prolene. His post-operative period was uneventful. Histo pathological report was consistent with intussusception. Patient was discharged on $20^{\text {th }}$ POD.

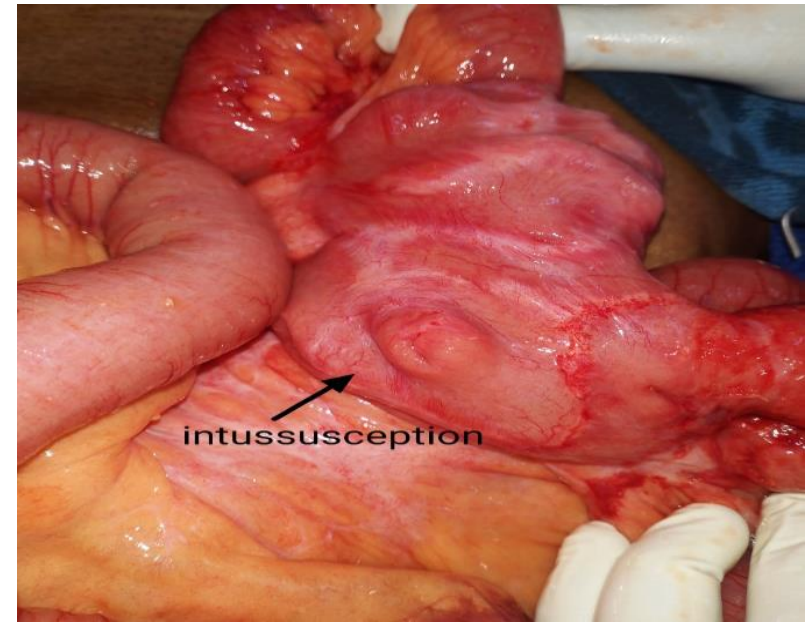

Figure 3: Intussusception.

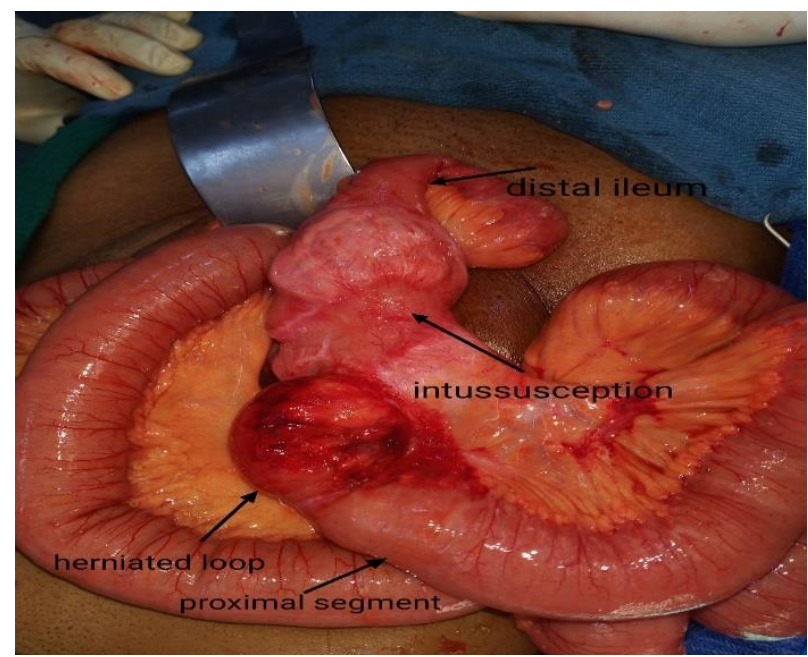

Figure 4: Picture showing both herniated loop and intussusception after reducing the contents.

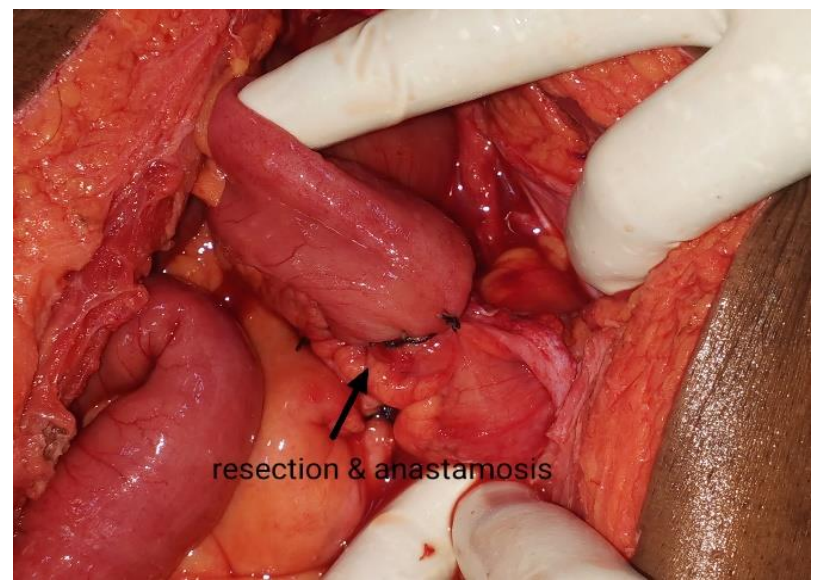

Figure 5: Post resection with anastomosis.

\section{DISCUSSION}

Internal hernia is defined as protrusion of a viscous through a peritoneal (most commonly visceral 
peritoneum) or mesenteric defect. ${ }^{2}$ Hypothesis regarding the pathogenesis of the pericecal fossa includes disruption of the normal process of intestinal rotation during embryonic development, tissue fragility due to aging, pressure elevation of the inner abdomen, retroperitoneal adhesion, post-abdominal surgery and vascular changes more common in old age. ${ }^{3}$

They may be congenital or acquired. Its incidence is less than $1 \%$. But the diagnosis becomes difficult when it comes to internal hernia as most of the cases presents as intestinal obstruction unlike the external hernia which presents initially as swelling. Acquired causes include post-surgical (e.g., roux-en-Y gastric bypass surgery or liver transplantation), trauma, peritoneal inflammation or ischemic changes. ${ }^{4}$ Congenital internal hernia usually are very rare. Small bowel is the most common content however mesentery can be involved. Its early prompt diagnosis and surgical intervention is needed as if left untreated leads to $>50 \%$ mortality.

Congenital internal hernias are further divided into retroperitoneal or congenital anomalous opening which lacks true peritoneal sac. Retro peritoneal hernias are classified into 1 . paraduodenal $(30-50 \%)$ most common, 2. foramen of Winslow also called as blandin hernia (6$10 \%), 3$. pericaecal $(8-12 \%), 4$. intersigmoid (4-8\%), 5 . pelvic and 6. para vesicle $(<6 \%)$. Anomalous opening include 1. transmesentric (8-10\%), 2. broad ligament (4$7 \%), 3$. transomental $(1-4 \%){ }^{4}$

Pericaecal hernias are responsible for $0.1-6.6 \%$ of internal hernias. Pericaecal hernia is a rare cause of small bowel obstruction. ${ }^{5}$ They tend to affect the adult population more commonly but also may occur in the paediatric age group. Pericaecal fossa is located behind the caecum and ascending colon and is limited by the parietocaecal fold outward and the mesentrico caecal fold inward. ${ }^{6}$ Pericaecal hernia may develop in 4 areassuperior ileo caecal, inferior ileocaecal, retrocaecal or paracolic. ${ }^{6}$ Superior ileocaecal hernia occurs in superior ileocaecal foassa which is formed by the fold in superior ileocolic mesentry and it contains anterior branch of ileocolic artery. ${ }^{6}$ Here the sac travels under right mesocolon. Inferior ileoceacal fossa has a prominent anterior ileoappendicular fold and it contains appendicular artery. Here the sac is under caecum. Retrocaecal hernia (hernia of rieux) is caused by partial defect of fixation of the right ascending mesocolon (toldt fascia) the content is between peritoneum on dorsal side, caecum ventrally and right ascending mesocolon superiorly. ${ }^{6}$

The paracaecal hernia that we are describing was due to herniation of the ileum through a congenital defect in the parietocaecal fold.

Patients may be asymptomatic or present with clinical symptoms of obstruction as the most frequently herniated organ is the small intestine. Intestinal ischemia and late cases of perforation presents to us as acute abdomen. Symptom severity relates to duration and reducibility of the hernia and the presence or absence of incarceration and strangulation. Clinical presentation is nonspecific making diagnosis, a challenge preoperatively. This leads diagnostic delays and increases in rates of ischemia, gangrene, and bowel resection. ${ }^{7}$

Computed tomography (CT) at present plays an important role in the evaluation of intestinal obstruction and acute abdomen. Both X-ray and ultrasonography are poor in detecting the etiology of intestinal obstruction. Multidetector CT can identify the specific site and severity of obstruction (partial versus complete), closed loop, and multiple segments of obstruction. It determines the etiology as well by identifying internal hernias and extra luminal lesions, such as masses, adenopathy, soft tissue infiltration, and vascular anomalies. ${ }^{7}$

Timely surgical intervention based on clinical suspicion and CT scan findings is necessary for the management of IH presenting with intestinal obstruction. Reduction of the strangulated intestinal segment should be done as early as possible to prevent intestinal ischemia, necrosis, and perforation and thereby reduce resection rates. Hernia defects should be closed with nonabsorbable sutures in order to prevent recurrence of internal herniation through the same orifices in the future. Recently laparoscopic technique has also been found to be useful for diagnosis and treatment of intestinal obstruction. ${ }^{7}$

\section{CONCLUSION}

Paracaecal hernia are rare type of pericaecal hernia which itself is a rare among internal hernias. They usually presents as intestinal obstruction. In present case also it presents with intestinal obstruction but intraoperatively it was caused by ileo ileal intussusception which was just proximal to herniated distal ileal loop. Intussusception was previously noted in paraduodenal type. But in paracaecal type, it's very rare. Internal hernias which when presents it may be due to obstruction, strangulation or incarceration. Immediate surgery is necessary to relieve the obstruction. Preoperative imaging is less conclusive in finding internal hernias. Hence in small bowel obstruction, internal hernias should be kept in mind.

\section{Funding: No funding sources Conflict of interest: None declared Ethical approval: Not required}

\section{REFERENCES}

1. Jang EJ, Cho SH, Kim DD. A case of small bowel obstruction due to a paracecal hernia. J Korean Soc Coloproctol. 2011;27(1):41-3.

2. Martin LC, Merkle EM, Thompson WM. Review of internal hernias: radiographic and clinical findings. Am J Roentgenol. 2006;186:703-17. 
3. Ogami T, Honjo H, Kusanagi H. Pericecal hernia manifesting as a small bowel obstruction successfully treated with laparoscopic surgery. J Surg Case Rep. 2016;2016(3):1-4.

4. Edwards HM, Al-Tayar H. A transmesenteric congenital internal hernia presenting in an adult. J Surg Case Rep. 2013;2013(12):rjt099.

5. AlJaberi LM, Salameh AK, Mashalah RM, AbuMaria A. Pericecal hernia in a pediatric patient: case report and literature review. Int $\mathbf{J}$ Surg Case Rep. 2019;60:296-8.
6. Takeyama N, Gokan T, Ohgiya Y, Satoh S, Hashizume T, Hataya K, et al. CT of internal hernias. Radiographics. 2005;25(4):997-1015.

7. Kar S, Mohapatra V, Rath PK. A rare type of primary internal hernia causing small intestinal obstruction. Case Rep Surg. 2016; 2016.

Cite this article as: Kumar MSK, Shyamsundar R, Girieasan SM, Kannan R, Nedunchezhiyan S. Rare case of paracaecal hernia with intussusception presenting as small bowel obstruction: case report. Int Surg J 2020;7:1662-5. 